\title{
Microengineered synthetic cellular microenvironment for stem cells
}

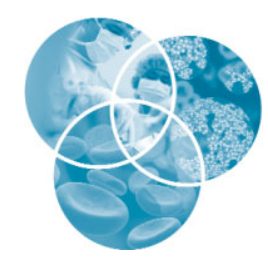

\author{
Yubing Sun, ${ }^{1}$ Shinuo $\mathrm{Weng}^{1}$ and Jianping Fu${ }^{1,2 *}$
}

\begin{abstract}
Stem cells possess the ability of self-renewal and differentiation into specific cell types. Therefore, stem cells have great potentials in fundamental biology studies and clinical applications. The most urgent desire for stem cell research is to generate appropriate artificial stem cell culture system, which can mimic the dynamic complexity and precise regulation of the in vivo biochemical and biomechanical signals, to regulate and direct stem cell behaviors. Precise control and regulation of the biochemical and biomechanical stimuli to stem cells have been successfully achieved using emerging micro/nanoengineering techniques. This review provides insights into how these micro/nanoengineering approaches, particularly microcontact printing and elastomeric micropost array, are applied to create dynamic and complex environment for stem cells culture. ๑ 2012 Wiley Periodicals, Inc.
\end{abstract}

How to cite this article:

WIREs Nanomed Nanobiotechnol 2012, 4:414-427. doi: 10.1002/wnan.1175

\section{INTRODUCTION}

$\mathrm{R}$ ecent advances in the identification, isolation, maintenance, and differentiation of stem cells, including somatic stem cells, embryonic stem cells (ESCs), and induced pluripotent stem cells (iPSCs), have opened exciting new avenues for developing stem cell-based therapies for various degenerative diseases and generating model systems for dissecting developmental processes and high-throughput drug screening. ${ }^{1-5}$ All these stem cell-based prospective biological and biomedical applications rely on maintaining and differentiating stem cells properly in vitro in a large scale and for a long term. However, maintaining undifferentiated stem cells and effectively controlling their differentiation in vitro are still among the greatest challenges that need to be addressed before stem cell-based applications can be realized for biomedical and clinical applications such as functional tissue engineering and regenerative medicine. . $^{6,7}$

In recent years, stem cell and developmental biologists have started to unravel the intricate molecular circuitry and intracellular signaling pathways

\footnotetext{
*Correspondence to: jpfu@umich.edu

${ }^{1}$ Department of Mechanical Engineering, Integrated Biosystems and Biomechanics Laboratory, University of Michigan, Ann Arbor, MI, USA

${ }^{2}$ Department of Biomedical Engineering, University of Michigan, Ann Arbor, MI, USA
}

regulating stem cell behaviors. ${ }^{2,8,9}$ Interestingly, there is a concurrent increased awareness in stem cell biology on the importance of the local cellular microenvironment through which various signaling cascades are regulated and integrated to control the fate decision of stem cells. ${ }^{10-12}$ As shown in Figure 1, soluble factors (e.g., growth factors and cytokines) and insoluble biophysical cues (e.g., cellular interactions with the surrounding extracellular matrix (ECM), cell-cell contacts with neighbouring and support cells, and interstitial flows) in the cellular microenvironment are both critically involved in the regulatory pathway for the stem cell fate decision. ${ }^{7,13,14}$ As an example, the quiescent hematopoietic stem cells (HSCs), which reside in the bone marrow, can be activated to differentiate into blood and immune cells. The niche cells for HSCs, primarily osteoblasts, can regulate intracellular signaling pathways in HSCs (such as Notch, Wingless (Wnt), Sonic hedgehog (Shh), and Smad pathways) critical for the fate decision of HSCs through either direct cell-cell contacts or paracrine signaling. ${ }^{15,16}$

Recently, with increased understanding of mechanobiology and signal transduction, the role of insoluble biophysical factors in the fate determination of stem cells is receiving accumulating attentions. ${ }^{17-20}$ The mechanosensory machinery of stem cells can sense and respond to mechanical stimuli from the local cellular microenvironment and relay these signals to the cell nuclei by recruiting kinases and other 


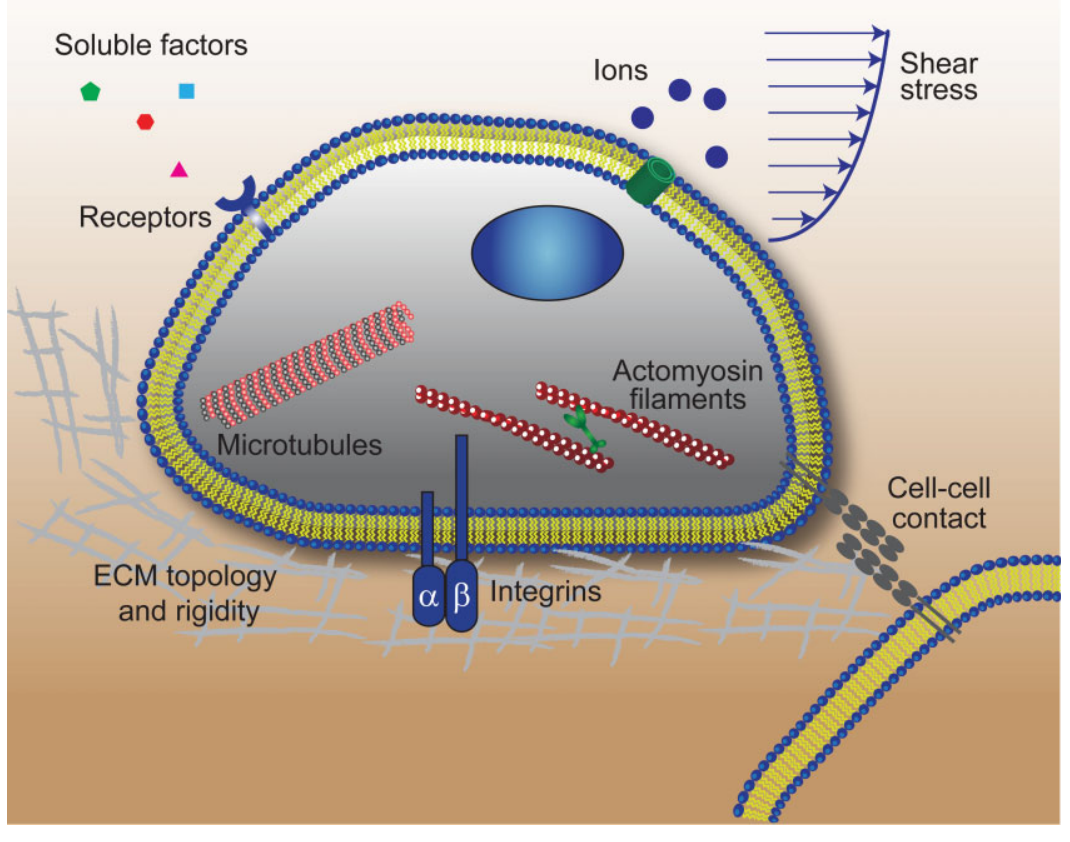

FIGURE 1 | Schematic showing the stem cell niche where interactions between stem cells and their local microenvironment regulate stem cell fate. The stem cell niche is a complex, dynamically regulated three-dimensional (3D) microenvironment comprising of soluble biochemical and insoluble biomechanical cues, adhesive signals as well as signals arising from direct cell-cell contacts. Soluble biochemical cues include small ions, growth factors, and cytokines, etc. Insoluble biophysical signals consist of matrix rigidity and topology, fluid shear stress, and other mechanical forces exerted by adjacent cells or owing to tissue growth and loading. Stem cells sense and respond to these biophysical stimuli through different mechanosensory components including heterodimeric integrins, mechanosensitive ion channels, cytoskeleton structures (actin microfilaments, microtubules, and intermediate filaments), and cell-cell contacts. signaling molecules to activate or inhibit transcription factors important for stem cell fate decisions. ${ }^{21,22}$ This mechanochemical conversion process is known as mechanotransduction and has been demonstrated to play a pivotal role in regulating cell adhesion, differentiation, migration, and apoptosis, etc. ${ }^{23-26}$ For cell-ECM interactions, activation of integrins upon binding to ECM proteins depends on the elastic modulus of the ECM and the local forces transmitted through cell adhesion sites (or focal adhesions, FAs), regardless the forces generated either internally by intracellular cytoskeletal contractility or externally by mechanical forces. ${ }^{27-30}$ Mechanical forces at FAs will further regulate dynamic recruitments of structural proteins as well as kinases such as FA kinases (FAK) to FAs. ${ }^{26,31}$ These kinases can further regulate small GTPases and downstream signaling cascades such as MAPK, PI3K/Akt, and RhoA/ROCK pathways. ${ }^{26,31}$ Many of these pathways have been shown to overlap or crosstalk with important signaling pathways that control stem cell fate. ${ }^{20}$ For example, MAPK signaling has been demonstrated essential for maintenance of the self-renewal of human ESCs (hESCs). ${ }^{32}$ Cell-cell contact mediated through cadherin is also considered as a driving force for tissue segregation, morphogenesis, and sorting of embryonic tissues. ${ }^{33,34}$ Interestingly, endogenous contractile force, or cell-cell tugging force, at the adherens junctions (AJs) has been shown to correlate positively with the AJ size. ${ }^{35}$ A more recent study has further shown that $\alpha$ catenin, which binds to E-cadherin, can serve as a stretch-activated tension sensing protein in the protein complex between E-cadherin and actomyosin structure. ${ }^{36}$ The crosstalk between cadherin activity and actomyosin cytoskeletal structure has been reported important for regulating the pluripotency of hESCs. ${ }^{37}$ Taken together, these studies strongly suggest the mechanosensitive properties of cell-ECM interactions and cell-cell contacts and their close relationship with the stem cell fate decision.

Collectively, the local microenvironment of stem cells in vivo is composed of complex dynamic interactions of biochemical and biomechanical cues, the dynamic and spatiotemporal regulation of which is critical for regulation of stem cell fate. However, conventional stem cell culture tools, which largely rely on static two-dimensional (2D) tissue culture plastic, is difficult, if not impossible, to mimic the dynamic complexity of the in vivo cellular microenvironment. To address this challenge, over the past decade, stem cell biologists and tissue engineering scientists have adapted and developed different novel and integrated micro/nanoengineering tools and devices for precise control and measurements of the cellular microenvironment at the cellular and subcellular resolutions. These micro/nanoscale tools and devices have been shown to provide powerful solutions for precise control of the ex vivo stem cell microenvironment. ${ }^{38-40}$ Using these micro/nanoscale devices and tools, exciting new biological insights have been generated as regard to the regulatory roles of the dynamic interactions of biochemical 
and biomechanical cues in regulating stem cell behaviors. $^{22,41-44}$

The major goal of this review is thus to offer a perspective on the new trend of designing artificial synthetic stem cell microenvironment using micro/nanotechnologies and their promise for future stem cell-based research and applications. In particular, we will review two widely used micro/nanotechnologies, i.e., microcontact printing (Figure 2) and elastomeric micropost array (Figure 3), and their applications for precise control of cell-ECM and cell-cell interactions at the cellular and subcellular levels in stem cell research. There are some other well-established micro/nanotechnologies, such as microfluidics (Figure 4), ${ }^{6,45-47}$ nanolithography, ${ }^{48-51}$ and stereolithography ${ }^{52-54}$ that have been applied successfully to control cellular and subcellular environmental cues to regulate and direct stem cell behaviors. These topics have been covered in some excellent reviews published elsewhere, ${ }^{6,7,40}$ where readers interested in further discussions in these topics can be referred to. Finally, we offer some speculations as to research directions and potential opportunities for developing micro/nanoscale tools and systems with new functionalities for controlling cell-ECM and cell-cell interactions for stem cell research.

\section{MICROCONTACT PRINTING TO CONTROL CELL-ECM INTERACTIONS}

\section{Introduction}

Early studies to control cell-ECM interactions took advantage of the observation that spreading of adherent mammalian cells could be regulated by molecular coating on cell culture surfaces. In an early study, Folkman and Moscona applied different concentrations of poly(2-hydroxyethyl methacrylate) (pHEMA) to cell culture surfaces to control cell spreading. This early study by Folkman and Moscona demonstrated that changes in the degree to which cells spread and flatten against a substrate appear to be tightly coupled to DNA synthesis and thus cellular growth control. ${ }^{64}$ In a follow-up study, Ingber and Folkman applied nonadhesive dishes coated with ECM proteins at varying densities to control cell spreading. ${ }^{65}$ Ingber and Folkman showed that cell spreading and size might be an inducer for the growth-differentiation switch of endothelial cells during angiogenesis. In another study, O'Neill et al. masked cover glasses with a patterned nonadhesive layer of pHEMA to constrain cell size. O'Neill et al. demonstrated that cell size could exert a direct effect on the cytoskeletal structure and cell-ECM interactions to regulate cellular growth. ${ }^{66}$
Even though these early studies supported the regulatory role of cell shape and size in controlling behaviors of adherent cells through their direct effects on intracellular cytoskeletal structures and cell-ECM interactions, interpretations of the experimental results reported in these studies sometimes could be complicated owing to the fact that in addition to changes in cell shape and size, changing molecular coating could also directly affect integrin ligation and thus cell-ECM interactions and adhesion-mediated signaling. In addition, these methods could require expensive and time-consuming sample preparation.

Microcontact printing has been developed over the last two decades as a high-throughput and precise techniques to control the cell-adhesive environment ${ }^{67,68}$ It is a rapid and affordable approach for substrate preparation to control cell shape and size as well as cell-ECM interactions without altering other chemical or mechanical cues in the cellular microenvironment. It has been widely used by cell biologists and tissue engineers to advance our understanding of how adherent stem cells interact with adhesive surfaces.

\section{Microcontact Printing for Stem Cell Research}

Microcontact printing is a soft lithography-based surface coating technique, which uses a relief elastomeric stamp made usually in poly(dimethylsiloxane) (PDMS) to transfer microscale patterns of silanes, alkanethiolates, and adhesive proteins from the stamp to cell culture surfaces (Figure 2(a)). Microcontact printing was originally developed by Whitesides and his colleagues in the early 1990s to control protein adsorption on cell culture surfaces ${ }^{67}$ and was later applied for patterning adhesion properties of mammalian cells. ${ }^{68}$

The original microcontact printing method for controlling cell-ECM interactions uses an elastomeric stamp to transfer patterns of functionalized long-chain alkanethiolates onto a gold-coated surface, where alkanethiolates coordinate and form a self-assembled monolayer (SAM) with the functional groups exposed to the solution. ${ }^{68-71}$ Using alkanethiolates with different terminal functionalities has provided a simple means to either resist or promote protein adsorption. For instance, hydrophobic SAMs adsorb proteins and can thus promote cell adhesion, while SAMs that terminate in ethylene glycol moieties resist protein adsorption and therefore prevent cell adhesion. To provide a more quantitative control over integrin ligation, investigators have developed methods to produce alkanethiolates terminated with adhesive 




FIG URE 2 | Microcontact printing to pattern adhesive protein islands. (a) Schematic drawing of standard procedures for stamp-on (left) and stamp-off (right) methods. (b) Deformations of poly(dimethylsiloxane) (PDMS) stamp during microcontact printing. Such deformations include lateral collapse (top) and sagging (bottom) of the PDMS stamp. (Reprinted with permission from Ref 55. Copyright 2007 Royal Society of Chemistry) (c) Representative fluorescence images of arrays of adhesive islands patterned using stamp-on (top) or stamp-off (bottom) methods. The squared-shaped arrays of $20 \mu \mathrm{m}$ adhesive islands are spaced 20 (left), 110 (middle), and $200 \mu \mathrm{m}$ (right) apart (edge-to-edge). (Reprinted with permission from Ref 56. Copyright 2011 Royal Society of Chemistry) (d) Left: Immunofluorescence image showing a single hMSC cultured on a flower-shaped adhesive island. The cell was costained for F-actin (green), vinculin (red) and nuclei (DAPl; blue). (Reprinted with permission from Ref 57. Copyright 2010 National Academy of Sciences USA) Right: Brightfield micrograph of a squared-shaped hMSC colony costained for alkaline phosphatase activity (ALP, blue) and lipid droplet accumulation (Lip, red) after 14 days of culture in a bipotential differentiation medium supportive for both osteogenic and adipogenic differentiation of hMSCs. (Reprinted with permission from Ref 58. Copyright 2008 John Wiley \& Sons Ltd.)

peptides, such as Arg-Gly-Asp (RGD), to control stem cell adhesion. ${ }^{72}$

While significant insights into regulation of cell-ECM interactions were obtained using microcontact printing with alkanethiolates, this method requires equipments for coating cell culture surfaces with gold and special chemicals, such as modified alkanethiolates; hence, microcontact printing with alkanethiolates is not readily adaptable for general use by the biological research community.

More recently, the advent of microcontact printing has addressed many of these shortcomings. Scientists have modified the microcontact printing process to facilitate cell patterning by directly stamping ECM proteins onto common cell culture substrates including glass, silicone rubber, and polystyrene. ${ }^{73,74}$ The 


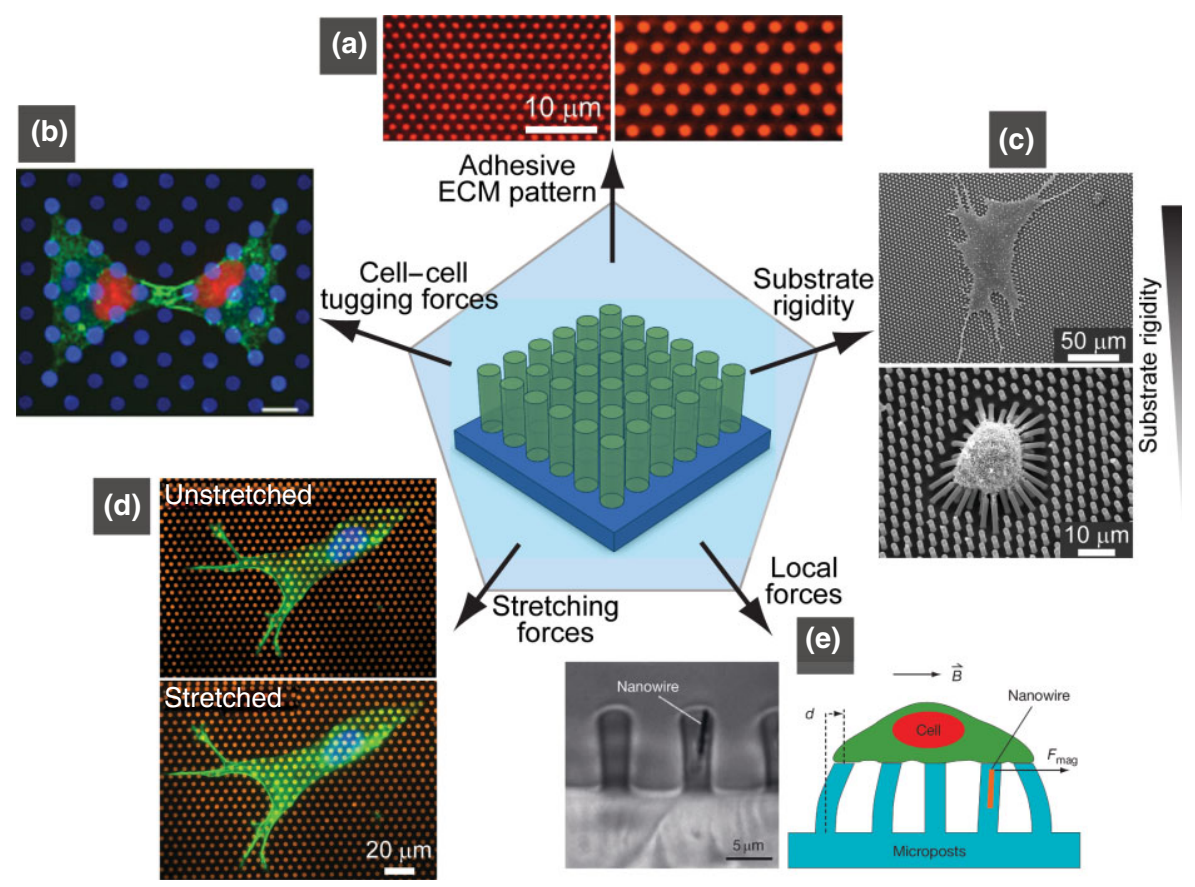

FIG URE 3 | Elastomeric poly(dimethylsiloxane) (PDMS) micropost array to control adhesive extracellular matrix (ECM) patterns, cell-cell contacts, substrate rigidity, cell stretching forces, and subcellular mechanical forces. (a) Modulating the PDMS micropost diameter and center-to-center spacing to regulate adhesive ECM patterns. (Reprinted with permission from Ref 59. Copyright 2011 Elsevier) (b) Cells constrained to a bowtie ECM pattern on the PDMS microposts using microcontact printing to regulate cell-cell contacts. Cells were costained for nuclei (DAPl; red) and AJs (green).

(Reprinted with permission from Ref 35. Copyright 2010 National Academy of Sciences USA) (c) Scanning electron microscopy images showing single human mesenchymal stem cells (hMSCs) plated on PDMS micropost arrays of the same surface geometry but different post heights to control substrate rigidity independently of effects on adhesive and other material surface properties. (Reprinted with permission from Ref 44 . Copyright 2010 Nature Publishing Group) (d) A stretchable micropost array membrane (mPAM) that could apply cell stretching forces to adherent cells attached on the micropost tops. (Reprinted with permission from Ref 60. Copyright 2012 Royal Society of Chemistry) (e) Magnetic PDMS microposts to apply local mechanical forces to adherent cells through individual focal adhesions. (Reprinted with permission from Ref 61. Copyright 2007 National Academy of Sciences USA)

improved microcontact printing process has become very convenient and has been successfully adapted in numerous biological studies to investigate effects of cell shape and cell-ECM interactions on cell functions. ${ }^{75-78}$ For example, using microcontact printing, Chen et al. showed that changes in the degree to which cells spread and flatten against a substrate could not only regulate cell proliferation but also apoptosis. ${ }^{71}$ Dike et al. used microcontact printing to study the geometric control of switching between growth, apoptosis, and differentiation of endothelial cells during angiogenesis. ${ }^{79}$ Théry et al. applied microcontact printing to demonstrate that cell adhesive microenvironment could govern cell internal organization and orientation of polarity. ${ }^{80,81}$

It is a more recent effort to use microcontact printing to study how cell-ECM interactions and cell shape regulate stem cell behaviors. In a landmark study, McBeath et al. reported that in response to a bipotential differentiation medium that contained inducers for both adipogenic and osteogenic differentiations of human mesenchymal stem cells (hMSCs), single hMSCs confined to small ECM islands selectively underwent adipogenesis, whereas single hMSCs on large ECM islands were biased toward osteogenesis. ${ }^{43}$ This osteogenicadipogenic switch in well-spread versus poorly spread hMSCs required generation of cytoskeletal tension through RhoA/ROCK-dependent actomyosin contractility. Another more recent study by Ruiz et al. confirmed the importance of cytoskeletal tension in regulating stem cell fate in the setting of multicellular structures. ${ }^{58}$ Ruiz et al. applied microcontact printing to control geometries of 2D structures of hMSCs. Ruiz et al. reported that in the presence of soluble factors permitting both osteogenic and adipogenic differentiations, hMSCs at the edge of the multicellular structures selectively differentiated into the osteogenic lineage, whereas those in the center became adipocytes.

Recently, Kilian et al. applied microcontact printing to demonstrate that in addition to the overall cell shape, cell geometry also plays an important 

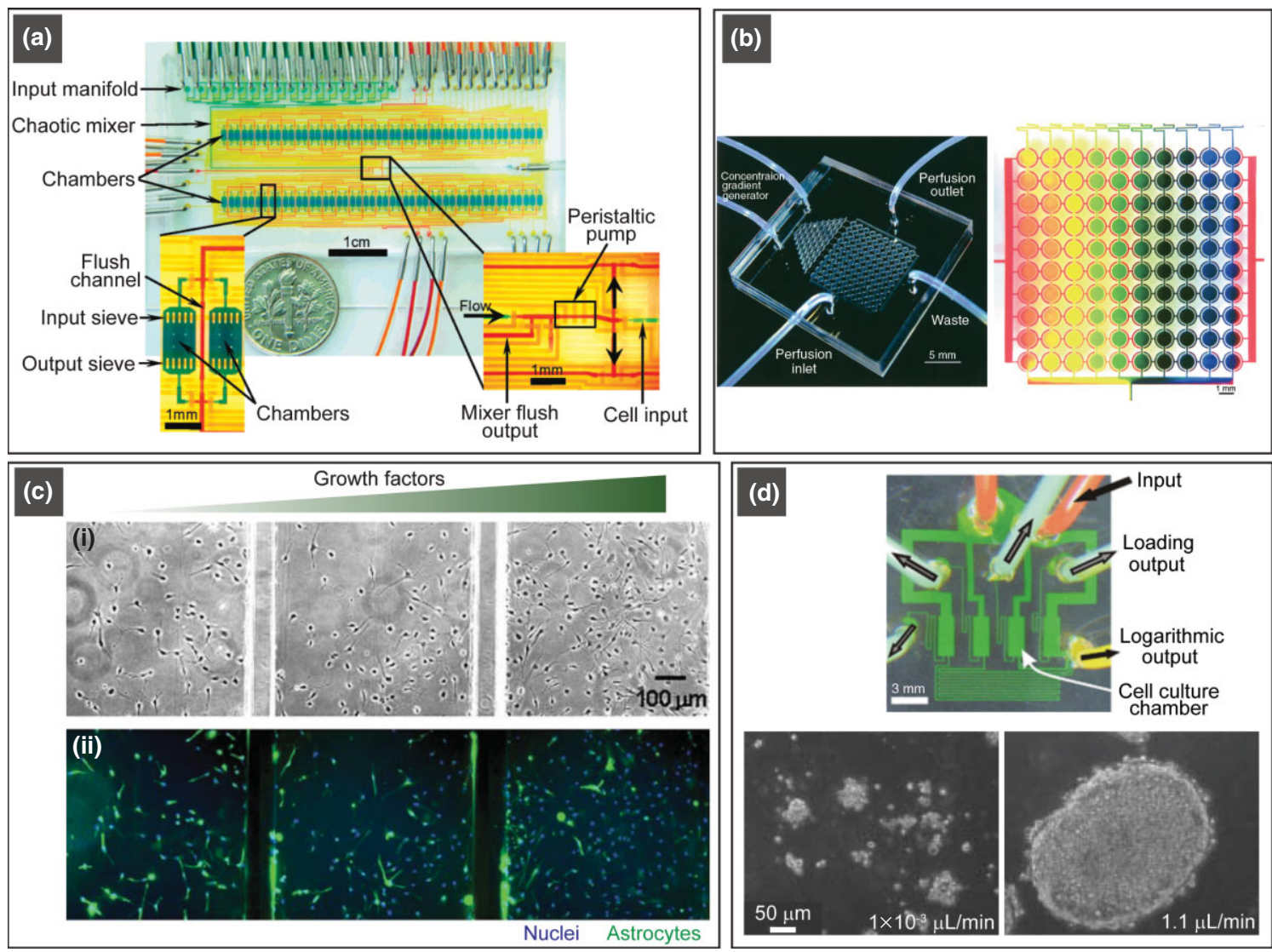

FIG URE 4 | Integrated microfluidic devices to control soluble biochemical cues and fluid shear stress to regulate stem cell function. (a) Design of a microfluidic chip for long-term stem cell culture. This fully automated cell culture screening microfluidic system could create arbitrary culture media formulations in 96 independent culture chambers and maintain stem cell viability for weeks. (Reprinted with permission from Ref 45 . Copyright 2007 American Chemical Society) (b) A microfluidic chemical gradient generator. (Reprinted with permission from Ref 62 . Copyright 2005 John Wiley \& Sons Ltd.) (c) Growth and differentiation of human neural stem cells (hNSCs) in a gradient-generating microfluidic device. hNSCs cultured in the gradient chamber for 7 days exhibited higher percentage of astrocyte differentiation (stained by an antibody against glial fibrillary acidic protein, an astrocyte marker; green) in the low growth factor region. (Reprinted with permission from Ref 63. Copyright 2005 Royal Society of Chemistry) (d) Microfluidic arrays for logarithmically perfused mouse embryonic stem cell (mESC) culture. The top photograph shows a microfluidic device fabricated using soft lithography with multiple chambers for long-term culture of $\mathrm{mESC}$. The bottom two Brightfield images show colonies of $\mathrm{mESC}$ after 4 days of perfusion at different culture flow rates. (Reprinted with permission from Ref 47. Copyright 2006 Royal Society of Chemistry)

role in regulating stem cell fate. ${ }^{57,82,83}$ Kilian et al. showed that in response to a bipotential differentiation medium that contained inducers for both the adipogenic and osteogenic differentiations, single hMSCs cultured in rectangles with increasing aspect ratio and in shapes with pentagonal symmetry but with different subcellular curvature-and with each occupying the same area-displayed different adipogenesis and osteogenesis profiles. Using cytoskeletaldisrupting pharmacological agents, Kilian et al. further confirmed a causal role for cytoskeletal tension in modulating the shape-based trends in lineage commitment of hMSCs.

Using microcontact printing, Peerani et al. demonstrated the effect of the cellular microenvironment on hESC fate by pattering hESC colonies onto defined adhesive islands with a controlled colony diameter. ${ }^{84}$ Peerani et al. showed that larger colonies with a high local cell density microenvironment would promote the maintenance of pluripotency in hESCs, through a niche size-dependent spatial gradient of BMP-mediated Smad1 signaling generated as a result of antagonistic interactions between hESCs and hESCderived extraembryonic endoderm. Thus, the effect of this colony size on the pluripotency maintenance of hESCs appears to be mediated by interactions among exogenously controlled parameters and autocrine and paracrine secretion of endogenously produced factors from hESCs.

More recently, using microcontact printing to define cell spread area, Connelly et al. demonstrated that the balance between self-renewal and 
differentiation of human epidermal stem cells depended on cell area. ${ }^{22}$ Connelly et al. found that confinement of the cell spread area using small ECM islands would trigger human epidermal stem cells to initiate terminal differentiation. Interestingly, this cell shape dependent differentiation of human epidermal stem cells did not depend on ECM composition or density, but was regulated by the actin cytoskeleton. Connelly et al. further discovered that the actin cytoskeleton-mediated shape-induced differentiation of human epidermal stem cells by regulating serum response factor (SRF) transcriptional activity. ${ }^{22}$

Taken together, the aforementioned studies demonstrate important roles of the cell-ECM interactions and cell shape and geometry in regulating stem cell fate. These studies highlight the complex interrelationships between the molecular events of cell adhesion (integrin ligation), the global changes in cytoskeletal structure and contractility, and activities of transcription factors to control gene expressions and stem cell behaviors. These studies further suggest the importance of understanding the regulatory relationship between these processes to advance our future models of adhesion-regulated stem cell function.

\section{Microcontact Printing Procedure and its Modification}

The common procedure for microcontact printing is as follows (Figure 2(a)). Microcontact printing uses elastomeric stamps containing the desired features to directly print chemistries or proteins onto cell culture substrates. Briefly, photolithography is used to generate an initial mold with an array of microscale features on a silicon wafer. A prepolymer of PDMS is then cured against this mold and peeled to reveal an elastomeric stamp containing the negative replica of the original mold. Stamps can then be inked with silanes, alkanethiols, or directly with ECM proteins. When the elastomeric stamp is placed in contact with a surface, the inked material is transferred to the receiving surface. Unstamped regions can be blocked by various substances that resist protein adsorption such as ethylene glycol-terminated alkanethiols or detergents (such as Pluronics F127 NF). When adherent cells are plated onto these surfaces, the cells will adhere selectively to the adhesive regions (those coated with ECM proteins) and will be blocked from attaching to or spreading into the nonadhesive regions.

It is evident that microcontact printing described above is a convenient and powerful method to define cell area and shape and control cell-ECM interactions. However, it suffers from a key limitation as the elastomeric stamp is deformable and thus elastomeric stamps bearing small, sparse features are prone to deformation, lateral collapsing, buckling, or sagging during stamping (Figure 2(b)). ${ }^{55}$ The elastomeric stamp collapse depends both on the geometry of the features, and the pressure applied during stamping. Recently, a modified microcontact printing method termed 'microcontact stamp-off' was developed by Desai et al. to specifically address this issue. ${ }^{56}$ In the microcontact stamp-off, a stamp with identical but inverse features is used to remove adsorbed ECM proteins from another topographically featureless stamp. This topographically featureless stamp is then placed in conformal contact with cell culture substrates to transfer the protein pattern. The microcontact stampoff method is an improved microcontact printing technique that can obviate the risk of pattern fouling associated with PDMS stamp deformation and collapse and hence can broaden the range of patterns that can be stamped using microcontact printing (Figure 2(c)).

\section{ELASTOMERIC MICROPOSTS FOR MECHANICAL REGULATION OF CELL FUNCTIONS}

\section{Introduction}

While microcontact printing can be applied efficiently to control cell adhesive microenvironment, biophysical signals including matrix mechanics and external mechanical forces are also critical regulatory cues in the local stem cell microenvironment. Matrix mechanics such as the elastic modulus of the ECM has been recently shown critical in regulating the self-renewal and differentiation of stem cells. ${ }^{85,86} \mathrm{Cel}-$ lular rigidity sensing involves intracellular cytoskeletal structures and actomyosin contractility. Thus, there is a great desire for stem cell culture platforms that can be conveniently used to modulate substrate rigidity while simultaneously measuring cellular traction forces exerted by stem cells.

Conventional methods to modulate substrate rigidity while simultaneously measuring cellular traction forces use synthetic hydrogels (such as polyacrylamide gel) with fluorescent microbeads embedded on the gel surface. ${ }^{87}$ The bulk rigidity of synthetic hydrogels can be tuned by modulating the amount of crosslinker, and cellular traction forces can be measured by mapping relative displacements of the microbeads before and after cell seeding (traction force microscopy, or TFM). ${ }^{88}$ Although this synthetic hydrogel-based approach has proven useful in characterizing $2 \mathrm{D}$ rigidity sensing and cell-ECM interactions, it has some intrinsic limitations, as altered crosslinker amount in hydrogels impacts not 
only their bulk mechanics but also molecular-scale material properties including porosity, surface chemistry, backbone flexibility and binding properties of immobilized adhesive ligands. ${ }^{89}$ TFM is also a computationally intensive, ill-posed method for calculating cellular traction forces from the displacement field of microbeads. Moreover, to achieve stable unique solutions from TFM, regularization schemes must be implemented, such as restricting traction forces to specific sites of adhesion and imposing constraints on the deformation field of the cell. Recently, Legant et al. has extended the application of TFM to threedimensional matrix with a computational method not restricted to specific sites of adhesions and the regularization schemes employed by this computational method remove all ill-posedness of the problem. ${ }^{88}$

Microfabricated elastomeric micropost arrays represent a drastically different approach to modulate substrate rigidity for cell culture while simultaneously measuring traction forces exerted by stem cells (Figure 3). ${ }^{90,91}$ Here, the elastomeric micropost array is fabricated using photolithography and replica molding with PDMS, and it consists of regularly spaced PDMS microposts. The mechanical properties of the PDMS micropost array are solely determined by its geometry and by Young's modules of PDMS. Thus, the substrate rigidity of the PDMS micropost array can be modulated simply by varying post height while keeping all other aspects of the substrate such as surface chemistry and ligand density unchanged. The PDMS microposts can be rendered adhesive by microcontact printing of adhesive proteins across micropost tops. Subsequent treatment with detergents (such as Pluronics F127 NF) to block nonspecific protein adsorption to the post shafts will further ensure that cells will only be able to adhere to the micropost tops and not crawl in between. Each PDMS micropost can function as an independent force sensor. ${ }^{90,91}$ Using fluorescence microscopy, the displacements of the bended micropost tops caused by local cellular traction forces can be measured, and thus traction forces can be quantified at a subcellular resolution. Another critical advantage of the PDMS micropost array is that measured traction forces can be attributed directly to distinct FAs, enabling mapping contractile forces to individual adhesions. ${ }^{90}$

Since its invention, the PDMS micropost array has been broadly applied to investigate mechanical forces involved in different biological systems (Figure 3). Notable examples include using the PDMS micropost array to study force-regulated adhesion formation, ${ }^{90}$ mechanics-mediated directional cell migration (mechanotaxis or durotaxis), ${ }^{92}$ contractile forces transmitted through cell-cell contacts (i.e., cell-cell tugging forces) ${ }^{35}$ (Figure 3(b)), mechanics of leukocyte transmigration through an endothelial monolayer, ${ }^{93,94}$ and clotting forces involved in thrombus formation. ${ }^{95}$ Given the close relevance of substrate rigidity and mechanical forces in regulating stem cell function, in this section we will focus on discussing using the PDMS micropost array to modulate substrate rigidity for stem cell culture and apply local and global mechanical forces to mediate mechanoresponsive behaviors of stem cells.

\section{Geometrically Modulated PDMS Microposts to Regulate Substrate Rigidity}

The spring constant $K$ of the PDMS micropost can be calculated using the Euler-Bernoulli theory as $K=$ $3 \pi \mathrm{ED}^{4} /\left(64 L^{3}\right)$, where $D$ and $L$ are the post diameter and height, respectively, and $E$ is Young's modules of PDMS. Substrate rigidity of the PDMS micropost array can be further characterized using an equivalent bulk modulus $E_{\text {eff }}$ of an continuous elastic substrate, and $E_{\text {eff }}$ is expressed as $E_{\text {eff }}=27 \mathrm{ED}^{3} /\left(128 L^{3}\right) .{ }^{59}$ Thus, substrate rigidity of the PDMS micropost array is solely determined by the bulk Young's modulus $E$ of PDMS and the post geometrical parameters including post height and diameter. The PDMS micropost arrays reported so far in the literature span a more than 10,000-fold range of rigidity from 0.06 to $4,000 \mathrm{nN} / \mu \mathrm{m}$ (the equivalent bulk modulus $E_{\text {eff }}$ ranging from $10 \mathrm{~Pa}$ to $3 \mathrm{MPa}$ ), much broader than is currently achievable with natural or synthetic hydrogels. 59

To approach the opportunity of using geometrically modulated PDMS microposts to regulate substrate rigidity, we and others modulated the post height $L$ of the PDMS micropost array to regulate substrate rigidity independently of effects on the material surface properties (Figure 3(c)). ${ }^{44,91}$ Using the PDMS micropost array, we investigated mechanoresponsive behaviors of hMSCs and demonstrated that the rigidity of the PDMS micropost array could impact cell morphology, FA formation, and cytoskeletal contractility of hMSCs. ${ }^{44}$ More interestingly, rigidity of the PDMS micropost array could regulate stem cell lineage commitment. For hMSCs plated on the PDMS micropost arrays of different rigidities, when exposed to a bipotential differentiation medium supportive of both osteogenic and adipogenic differentiation, osteogenic lineage was favored for hMSCs on the rigid micropost arrays whereas adipogenic differentiation was enhanced on soft ones. The PDMS micropost array could be further utilized for live-cell measurements of endogenous cytoskeletal contractility of single hMSCs to predict the later onset of their differentiation. We 
showed that commitment of hMSCs in single cells could be predicted a priori by monitoring the early evolution of their contractile states during their lineage commitment process. Such early, noninvasive predictors of cell fate decisions might have utility in accelerating stem cell differentiation studies, for example, in the context of drug screening, diagnostics, and regenerative medicine.

Very recently, the PDMS micropost array was used by Weng and Fu to study the synergistic and independent effects of substrate rigidity and adhesive ECM pattern on mechanoresponsive behaviors of adherent cells (Figure 3(a)). Mechanoresponsive behaviors of NIH/3T3 fibroblasts and human umbilical vein endothelial cells (HUVECs), including cell spreading, FA formation, cytoskeletal contractility, and proliferation were all strongly dependent on both substrate rigidity and adhesive ECM pattern. Under the same substrate rigidity condition, smaller and closer adhesive ECM islands could cause both cells to spread out more, form more adhesion structures, and have a higher proliferation rate. The influence of adhesive ECM pattern on rigidity-mediated cytoskeletal contractility was cell type specific and was only significant for NIH/3T3. Thus, the results reported by Weng and Fu highlight the complex interrelationships between adhesion-mediated cell signaling and rigidity sensing, and understanding their relationship will be critical to advance our future models of adhesion- and mechanics-regulated stem cell function.

\section{Active Mechanical Force Application Using PDMS Microposts}

Aside from its applications for passively regulating substrate rigidity and reporting cellular traction forces, the PDMS micropost array has been integrated with actuation technologies as a means to both apply active forces to adherent cells and to simultaneously report dynamic cellular contractile responses. ${ }^{60,61}$ This approach is especially attractive for modulating stem cell behaviors, as studies have shown that applications of external forces to perturb the mechanosensory machinery of stem cells can result in their rapid functional responses. ${ }^{96-99}$

To generate active mechanical forces using the PDMS microposts, Sniadecki et al. embedded individual magnetic nanowires into the PDMS micropost (Figure 3(e)). ${ }^{61}$ Under a uniform magnetic field, the PDMS micropost embedded with magnetic nanowires could be actuated to apply local forces to individual FA sites. Consistent with prior reports, Sniadecki et al. observed that local force perturbation could trigger remodeling and reinforcement of FAs experiencing the forces. Interestingly, Sniadecki et al. also found that local forces could induce long-range relaxation of traction forces, even though the underlying mechanism is still largely unclear.

The PDMS micropost array embedded with magnetic nanowires is an attractive tool to study forcemediated FA dynamics and cell mechanics. However, it remains largely unclear whether the mechanical response of cells to the local force perturbation is spatially restricted to the subcellular region that experiences the force, or results in a global change in cellular mechanics. Results reported from others have suggested that the local force application methods can suffer from variability among the data from different studies, which is suspected to be due to the regional heterogeneity and dynamic regulation of the cytoplasm and adhesions of cells. ${ }^{100}$

Applying global cell stretching forces to adherent cells using the PDMS micropost array was recently achieved by Mann et al., who integrated the PDMS micropost array onto a stretchable PDMS membrane (Figure $3(\mathrm{~d})) .{ }^{60}$ Using a vacuum, the micropost array membrane (mPAM) system could be stretched to apply homogenous stretching forces to adherent cells attached on the PDMS micropost tops. Compared with conventional methods using continuous deformable membranes, the mPAM offered the unique advantage of a precise and independent control of both substrate rigidity and mechanical stretch, while simultaneously measuring live-cell responses of cytoskeletal contractility. Stretch-induced strain in mPAM was mostly constrained within the PDMS membrane and did not propagate up PDMS post. ${ }^{60}$ Thus, stretch of the mPAM would not affect micropost geometry and thus substrate rigidity. While for continuous membrane methods, stretch-induced strain-stiffening behavior in the membrane often is unavoidable, ${ }^{101,102}$ introducing an undesired potent mechanical signal known to affect cellular behaviors. Stretch of the mPAM would not generate mechanical strain directly in FAs on micropost tops, another critical difference from continuous membranes, which directly strain FAs during stretch. During mPAM stretch, the stretch amplitude could be easily determined in situ by utilizing the regularly positioned and undeflected posts as fiduciary markers to quantify their increased centerto-center distances under different levels of stretch.

Using the mPAM, Mann et al. studied the live-cell subcellular dynamic responses of contractile forces in vascular smooth muscle cells (VSMCs) to a sustained static equibiaxial cell stretch. ${ }^{60}$ Mann et al. showed that in response to a sustained cell stretch, VSMCs regulated their cytoskeletal contractility in a biphasic manner: they first acutely enhanced 
their contraction to resist rapid cell deformation ('stiffening') before they allowed slow adaptive inelastic cytoskeletal reorganization to release their contractility ('softening').

As discussed, the mPAM is a promising tool that can offer a precise and independent control of both substrate rigidity and mechanical stretch, while simultaneously measuring live-cell responses of cytoskeletal contractility. Previous studies indicated that mechanical stretch could promote the self-renewal of hESCs. ${ }^{97}$ Thus, it would be interesting to apply the newly developed mPAM system to study mechanics-mediated hESC function, including their self-renewal and directed differentiation.

\section{CONCLUSION AND FUTURE OUTLOOK}

Stem cells fate decisions are regulated closely by their local microenvironment, which is complex, dynamic, and heterogeneous. While biochemical factors play important roles in regulating the self-renewal and differentiation of stem cells, biomechanical cues such as ECM rigidity, external mechanical forces, and nanotopography are also pivotal and can trigger multiple signaling pathways to regulate the fate of stem cells. Different micro/nanoengineering techniques have been developed over the last decade or so to establish a synthetic cellular microenvironment to control the biochemical and biomechanical cues in a highly integrated and controlled manner. In particular, microcontact printing has been proven powerful to directly pattern the shape and size of single stem cells and stem cell colonies, which can directly impact the cytoskeletal structure and contractility of stem cells to regulate their behaviors. Microfabricated elastomeric micropost array is a promising system that can be adapted to achieve different functionalities such as modulating ECM rigidity, measuring traction forces, and applying local and global forces to stem cells. These micro/nanoengineering tools can be combined with other existing micro/nanoscale devices or techniques to provide a comprehensive control of the local cellular microenvironment for stem cells at the subcellular or cellular resolution to direct their behaviors.

Despite the current achievements using micro/ nanoengineering tools to generate well-controlled stem cell culture platforms, their applications toward large-scale stem cell culture are still limited. Most systems are still more suitable for single-cell analysis or high-throughput screening, rather than for scalable stem cell culture and production. In addition, these techniques are still far away from being broadly adapted in common biological laboratories, as applications and usages of these systems still require specific engineering expertises. Thus, future development of microengineered synthetic cellular microenvironment for stem cell research will require closer collaborations between stem cell and developmental biologists, tissue engineers, micro/nanoengineering scientists. In the future, it would be highly desirable to develop facile, standard and automatic systems using micro/nanoengineering techniques to control stem cell microenvironment.

\section{ACKNOWLEDGMENTS}

We acknowledge financial support from the National Science Foundation (CMMI 1129611), the National Institute of Health (UL1RR024986), and the department of Mechanical Engineering at the University of Michigan, Ann Arbor. The valuable input from all the members of the Integrated Biosystems and Biomechanics Laboratory is greatly appreciated. Finally, we extend our apologies to all our colleagues in the field whose work we were unable to cite formally because of the space limitation.

\section{REFERENCES}

1. Daley GQ, Scadden DT. Prospects for stem cell-based therapy. Cell 2008, 132:544-548.

2. Murry CE, Keller G. Differentiation of embryonic stem cells to clinically relevant populations: lessons from embryonic development. Cell 2008, 132:661-680.

3. Thomson JA, Itskovitz-Eldor J, Shapiro SS, Waknitz MA, Swiergiel JJ, Marshall VS, Jones JM. Embryonic stem cell lines derived from human blastocysts. Science 1998, 282:1145-1147.
4. Ebert AD, Svendsen CN. Human stem cells and drug screening: opportunities and challenges. Nat Rev Drug Discov 2010, 9:367-372.

5. Takahashi K, Yamanaka S. Induction of pluripotent stem cells from mouse embryonic and adult fibroblast cultures by defined factors. Cell 2006, 126:663-676.

6. Toh Y-C, Blagovic K, Voldman J. Advancing stem cell research with microtechnologies: opportunities and challenges. Integr Biol 2010, 2:305-325. 
7. Keung AJ, Kumar S, Schaffer DV. Presentation counts: microenvironmental regulation of stem cells by biophysical and material cues. Annu Rev Cell Dev Biol 2010, 26:533-556.

8. Jaenisch R, Young R. Stem cells, the molecular circuitry of pluripotency and nuclear reprogramming. Cell 2008, 132:567-582.

9. Pera MF, Tam PPL. Extrinsic regulation of pluripotent stem cells. Nature 2010, 465:713-720.

10. Scadden DT. The stem-cell niche as an entity of action. Nature 2006, 441:1075-1079.

11. Jones DL, Wagers AJ. No place like home: anatomy and function of the stem cell niche. Nat Rev Mol Cell Biol 2008, 9:11-21.

12. Morrison SJ, Spradling AC. Stem cells and niches: mechanisms that promote stem cell maintenance throughout life. Cell 2008, 132:598-611.

13. Lyssiotis CA, Lairson LL, Boitano AE, Wurdak H, Zhu S, Schultz PG. Chemical control of stem cell fate and developmental potential. Angew Chem Int Ed Engl 2011, 50:200-242.

14. Moore KA, Lemischka IR. Stem cells and their niches. Science 2006, 311:1880-1885.

15. Blank U, Karlsson G, Karlsson S. Signaling pathways governing stem-cell fate. Blood 2008, 111:492-503.

16. Holst J, Watson S, Lord MS, Eamegdool SS, Bax DV, Nivison-Smith LB, Kondyurin A, Ma L, Oberhauser AF, Weiss AS, et al. Substrate elasticity provides mechanical signals for the expansion of hemopoietic stem and progenitor cells. Nat Biotechnol 2010, 28:1123-1128.

17. Wolf CB, Mofrad MRK. Mechanotransduction and its role in stem cell biology. In: Baharvand H, ed. Trends in Stem Cell Biology and Technology. New York, NY: Humana Press; 2009, 389-403.

18. Titushkin IA, Shin J, Cho M. A new perspective for stem-cell mechanobiology: biomechanical control of stem-cell behavior and fate. Crit Rev Biomed Eng 2010, 38:393-433.

19. Discher DE, Mooney DJ, Zandstra PW. Growth factors, matrices, and forces combine and control stem cells. Science 2009, 324:1673-1677.

20. Sun Y, Chen CS, Fu J. Forcing stem cells to behave: a biophysical perspective of the cellular microenvironment. Annu Rev Biophys 2012, 41:23.1-23.24.

21. Dupont S, Morsut L, Aragona M, Enzo E, Giulitti S, Cordenonsi M, Zanconato F, Le Digabel J, Forcato M, Bicciato S, et al. Role of YAP/TAZ in mechanotransduction. Nature 2011, 474:179-183.

22. Connelly JT, Gautrot JE, Trappmann B, Tan DWM, Donati G, Huck WTS, Watt FM. Actin and serum response factor transduce physical cues from the microenvironment to regulate epidermal stem cell fate decisions. Nat Cell Biol 2010, 12:711-U177.
23. Chen CS. Mechanotransduction - a field pulling together? J Cell Sci 2008, 121:3285-3292.

24. Hoffman BD, Grashoff C, Schwartz MA. Dynamic molecular processes mediate cellular mechanotransduction. Nature 2011, 475:316-323.

25. Wang N, Tytell JD, Ingber DE. Mechanotransduction at a distance: mechanically coupling the extracellular matrix with the nucleus. Nat Rev Mol Cell Biol 2009, 10:75-82.

26. Geiger B, Spatz JP, Bershadsky AD. Environmental sensing through focal adhesions. Nat Rev Mol Cell Biol 2009, 10:21-33.

27. Lele TP, Pendse J, Kumar S, Salanga M, Karavitis J, Ingber DE. Mechanical forces alter zyxin unbinding kinetics within focal adhesions of living cells. J Cell Physiol 2006, 207:187-194.

28. Galbraith CG, Yamada KM, Sheetz MP. The relationship between force and focal complex development. J Cell Biol 2002, 159:695-705.

29. Riveline D, Zamir E, Balaban NQ, Schwarz US, Ishizaki T, Narumiya S, Kam Z, Geiger B, Bershadsky AD. Focal contacts as mechanosensors: externally applied local mechanical force induces growth of focal contacts by an Mdia1-dependent and Rock-independent mechanism. J Cell Biol 2001, 153: 1175-1186.

30. Balaban NQ, Schwarz US, Riveline D, Goichberg P, Tzur G, Sabanay I, Mahalu D, Safran S, Bershadsky A, Addadi L, et al. Force and focal adhesion assembly: a close relationship studied using elastic micropatterned substrates. Nat Cell Biol 2001, 3:466-472.

31. Parsons JT, Horwitz AR, Schwartz MA. Cell adhesion: integrating cytoskeletal dynamics and cellular tension. Nat Rev Mol Cell Biol 2010, 11:633-643.

32. Li J, Wang G, Wang C, Zhao Y, Zhang H, Tan Z, Song Z, Ding M, Deng H. MEK/ERK signaling contributes to the maintenance of human embryonic stem cell self-renewal. Differentiation 2007, 75:299-307.

33. Steinberg MS. Reconstruction of tissues by dissociated cells. Some morphogenetic tissue movements and the sorting out of embryonic cells may have a common explanation. Science 1963, 141:401-408.

34. Halbleib JM, Nelson WJ. Cadherins in development: cell adhesion, sorting, and tissue morphogenesis. Genes Dev 2006, 20:3199-3214.

35. Liu Z, Tan JL, Cohen DM, Yang MT, Sniadecki NJ, Ruiz SA, Nelson CM, Chen CS. Mechanical tugging force regulates the size of cell-cell junctions. Proc Natl Acad Sci 2010, 107:9944-9949.

36. Yonemura S, Wada Y, Watanabe T, Nagafuchi A, Shibata M. $\alpha$-Catenin as a tension transducer that induces adherens junction development. Nat Cell Biol 2010, $12: 533-542$

37. Li D, Zhou J, Wang L, Shin ME, Su P, Lei X, Kuang $\mathrm{H}$, Guo W, Yang $\mathrm{H}$, Cheng L, et al. Integrated biochemical and mechanical signals regulate multifaceted 
human embryonic stem cell functions. J Cell Biol 2010, 191:631-644.

38. Lutolf MP, Hubbell JA. Synthetic biomaterials as instructive extracellular microenvironments for morphogenesis in tissue engineering. Nat Biotechnol 2005, 23:47-55.

39. Khademhosseini A, Langer R, Borenstein J, Vacanti JP. Microscale technologies for tissue engineering and biology. Proc Natl Acad Sci U S A 2006, 103: 2480-2487.

40. Kshitiz, Kim DH, Beebe DJ, Levchenko A. Micro- and nanoengineering for stem cell biology: the promise with a caution. Trends Biotechnol 2011, 29:399-408.

41. Gilbert PM, Havenstrite KL, Magnusson KEG, Sacco A, Leonardi NA, Kraft P, Nguyen NK, Thrun S, Lutolf MP, Blau HM. Substrate elasticity regulates skeletal muscle stem cell self-renewal in culture. Science 2010, 329:1078-1081.

42. Przybyla LM, Voldman J. Attenuation of extrinsic signaling reveals the importance of matrix remodeling on maintenance of embryonic stem cell self-renewal. Proc Natl Acad Sci 2012, 109:835-840.

43. McBeath R, Pirone DM, Nelson CM, Bhadriraju K, Chen CS. Cell shape, cytoskeletal tension, and RhoA regulate stem cell lineage commitment. Developmental Cell 2004, 6:483-495.

44. Fu JP, Wang YK, Yang MT, Desai RA, Yu XA, Liu ZJ, Chen CS. Mechanical regulation of cell function with geometrically modulated elastomeric substrates. Nat Methods 2010, 7:733-736.

45. Gomez-Sjoberg R, Leyrat AA, Pirone DM, Chen CS, Quake SR. Versatile, fully automated, microfluidic cell culture system. Anal Chem 2007, 79:8557-8563.

46. Lecault V, Vaninsberghe M, Sekulovic S, Knapp DJ, Wohrer S, Bowden W, Viel F, McLaughlin T, Jarandehei A, Miller M, et al. High-throughput analysis of single hematopoietic stem cell proliferation in microfluidic cell culture arrays. Nat Methods 2011, 8: 581-586.

47. Kim L, Vahey MD, Lee HY, Voldman J. Microfluidic arrays for logarithmically perfused embryonic stem cell culture. Lab Chip 2006, 6:394-406.

48. Dalby MJ, Gadegaard N, Tare R, Andar A, Riehle MO, Herzyk P, Wilkinson CDW, Oreffo ROC. The control of human mesenchymal cell differentiation using nanoscale symmetry and disorder. Nat Mater 2007, 6:997-1003.

49. Yim EKF, Pang SW, Leong KW. Synthetic nanostructures inducing differentiation of human mesenchymal stem cells into neuronal lineage. Exp Cell Res 2007, 313:1820-1829.

50. Oh S, Brammer KS, Li YSJ, Teng D, Engler AJ, Chien S, Jin $S$. Stem cell fate dictated solely by altered nanotube dimension. Proc Natl Acad Sci 2009, 106:2130-2135.
51. McMurray RJ, Gadegaard N, Tsimbouri PM, Burgess KV, McNamara LE, Tare R, Murawski K, Kingham E, Oreffo ROC, Dalby MJ. Nanoscale surfaces for the long-term maintenance of mesenchymal stem cell phenotype and multipotency. Nat Mater 2011, 10: 637-644.

52. Seck TM, Melchels FP, Feijen J, Grijpma DW. Designed biodegradable hydrogel structures prepared by stereolithography using poly(ethylene glycol)/ poly(D,L-lactide)-based resins. J Control Release 2010, 148:34-41.

53. Chan V, Zorlutuna P, Jeong JH, Kong H, Bashir R. Three-dimensional photopatterning of hydrogels using stereolithography for long-term cell encapsulation. Lab Chip 2010, 10:2062-2070.

54. Zorlutuna P, Jeong JH, Kong H, Bashir R. Stereolithography-based hydrogel microenvironments to examine cellular interactions. Adv Funct Mater 2011, 21:3642-3651.

55. Ruiz SA, Chen CS. Microcontact printing: a tool to pattern. Soft Matter 2007, 3:168-177.

56. Desai RA, Khan MK, Gopal SB, Chen CS. Subcellular spatial segregation of integrin subtypes by patterned multicomponent surfaces. Integr Biol 2011, 3:560-567.

57. Kilian KA, Bugarija B, Lahn BT, Mrksich M. Geometric cues for directing the differentiation of mesenchymal stem cells. Proc Natl Acad Sci U S A 2010, 107:4872-4877.

58. Ruiz SA, Chen CS. Emergence of patterned stem cell differentiation within multicellular structures. Stem Cells 2008, 26:2921-2927.

59. Weng S, Fu J. Synergistic regulation of cell function by matrix rigidity and adhesive pattern. Biomaterials 2011, 32:9584-9593.

60. Mann JM, Lam RHW, Weng S, Sun Y, Fu J. A siliconebased stretchable micropost array membrane for monitoring live-cell subcellular cytoskeletal response. Lab Chip 2012, 12:731-740.

61. Sniadecki NJ, Anguelouch A, Yang MT, Lamb CM, Liu Z, Kirschner SB, Liu Y, Reich DH, Chen CS. Magnetic microposts as an approach to apply forces to living cells. Proc Natl Acad Sci U S A 2007, 104: 14553-14558.

62. Hung PJ, Lee PJ, Sabounchi P, Lin R, Lee LP. Continuous perfusion microfluidic cell culture array for high-throughput cell-based assays. Biotechnol Bioeng 2005, 89:1-8.

63. Chung BG, Flanagan LA, Rhee SW, Schwartz PH, Lee AP, Monuki ES, Jeon NL. Human neural stem cell growth and differentiation in a gradient-generating microfluidic device. Lab Chip 2005, 5:401-406.

64. Folkman J, Moscona A. Role of cell shape in growth control. Nature 1978, 273:345-349.

65. Ingber DE, Folkman J. Mechanochemical switching between growth and differentiation during fibroblast 
growth factor-stimulated angiogenesis in vitro: role of extracellular matrix. J Cell Biol 1989, 109:317-330.

66. O’Neill C, Jordan P, Riddle P, Ireland G. Narrow linear strips of adhesive substratum are powerful inducers of both growth and total focal contact area. J Cell Sci 1990, 95:577-586.

67. Prime K, Whitesides G. Self-assembled organic monolayers: model systems for studying adsorption of proteins at surfaces. Science 1991, 252:1164-1167.

68. Lopez GP, Albers MW, Schreiber SL, Carroll R, Peralta E, Whitesides GM. Convenient methods for patterning the adhesion of mammalian cells to surfaces using self-assembled monolayers of alkanethiolates on gold. J Am Chem Soc 1993, 115:5877-5878.

69. Singhvi R, Kumar A, Lopez G, Stephanopoulos G, Wang D, Whitesides G, Ingber D. Engineering cell shape and function. Science 1994, 264:696-698.

70. Spargo BJ, Testoff MA, Nielsen TB, Stenger DA, Hickman JJ, Rudolph AS. Spatially controlled adhesion, spreading, and differentiation of endothelial cells on self-assembled molecular monolayers. Proc Natl Acad Sci 1994, 91:11070-11074.

71. Chen CS, Mrksich M, Huang S, Whitesides GM, Ingber DE. Geometric control of cell life and death. Science 1997, 276:1425-1428.

72. Hudalla GA, Murphy WL. Using “click” chemistry to prepare sam substrates to study stem cell adhesion. Langmuir 2009, 25:5737-5746.

73. Tan J, Liu W, Nelson CM, Raghavan S, Chen CS. Simple approach to micropattern cells on common culture substrates by tuning substrate wettability. Tissue Eng 2004, 10:865-872.

74. Bernard A, Delamarche E, Schmid H, Michel B, Bosshard HR, Biebuyck H. Printing patterns of proteins. Langmuir 1998, 14:2225-2229.

75. Rape AD, Guo WH, Wang YL. The regulation of traction force in relation to cell shape and focal adhesions. Biomaterials 2011, 32:2043-2051.

76. Rottmar M, Håkanson M, Smith M, ManiuraWeber K. Stem cell plasticity, osteogenic differentiation and the third dimension. J Mater Sci: Mater Med 2010, 21:999-1004.

77. Feinberg AW, Feigel A, Shevkoplyas SS, Sheehy S, Whitesides GM, Parker KK. Muscular thin films for building actuators and powering devices. Science 2007, 317:1366-1370.

78. Anderson D, Hinds M. Endothelial cell micropatterning: methods, effects, and applications. Ann Biomed Eng 2011, 39:2329-2345.

79. Dike L, Chen C, Mrksich M, Tien J, Whitesides G, Ingber D. Geometric control of switching between growth, apoptosis, and differentiation during angiogenesis using micropatterned substrates. In Vitro Cell Dev Biol - Animal 1999, 35:441-448.

80. Théry M, Racine V, Piel M, Pépin A, Dimitrov A, Chen Y, Sibarita J-B, Bornens M. Anisotropy of cell adhesive microenvironment governs cell internal organization and orientation of polarity. Proc Natl Acad Sci 2006, 103:19771-19776.

81. Théry M, Pépin A, Dressaire E, Chen Y, Bornens M. Cell distribution of stress fibres in response to the geometry of the adhesive environment. Cell Motil Cytoskel 2006, 63:341-355.

82. Luo W, Jones SR, Yousaf MN. Geometric control of stem cell differentiation rate on surfaces. Langmuir 2008, 24:12129-12133.

83. Song W, Lu HX, Kawazoe N, Chen GP. Adipogenic Differentiation of individual mesenchymal stem cell on different geometric micropatterns. Langmuir 2011, 27:6155-6162.

84. Peerani R, Rao BM, Bauwens C, Yin T, Wood GA, Nagy A, Kumacheva E, Zandstra PW. Niche-mediated control of human embryonic stem cell self-renewal and differentiation. EMBO J 2007, 26:4744-4755.

85. Discher DE, Janmey P, Wang Y-L. Tissue cells feel and respond to the stiffness of their substrate. Science 2005, 310:1139-1143.

86. Engler AJ, Sen S, Sweeney HL, Discher DE. Matrix elasticity directs stem. Cell 2006, 126:677-689.

87. Dembo M, Wang Y-L. Stresses at the cell-to-substrate interface during locomotion of fibroblasts. Biophys J 1999, 76:2307-2316.

88. Legant WR, Miller JS, Blakely BL, Cohen DM, Genin GM, Chen CS. Measurement of mechanical tractions exerted by cells in three-dimensional matrices. Nat Methods 2010, 7:969-971.

89. Wong JY, Leach JB, Brown XQ. Balance of chemistry, topography, and mechanics at the cell-biomaterial interface: issues and challenges for assessing the role of substrate mechanics on cell response. Surf Sci 2004, 570:119-133.

90. Tan JL, Tien J, Pirone DM, Gray DS, Bhadriraju K, Chen CS. Cells lying on a bed of microneedles: An approach to isolate mechanical force. Proc Natl Acad Sci U S A 2003, 100:1484-1489.

91. Saez A, Buguin A, Silberzan P, Ladoux B. Is the mechanical activity of epithelial cells controlled by deformations or forces? Biophys J 2005, 89:L52-L54.

92. du Roure O. Force mapping in epithelial cell migration. Proc Natl Acad Sci U S A 2005, 102:2390-2395.

93. Rabodzey A, Alcaide P, Luscinskas FW, Ladoux B. Mechanical forces induced by the transendothelial migration of human neutrophils. Biophys J 2008, 95:1428-1438.

94. Liu Z, Sniadecki N, Chen C. Mechanical forces in endothelial cells during firm adhesion and early transmigration of human monocytes. Cell Mol Bioeng 2010, 3:50-59.

95. Liang XM, Han SJ, Reems J-A, Gao D, Sniadecki NJ. Platelet retraction force measurements using flexible post force sensors. Lab Chip 2010, 10:991-998. 
96. Chowdhury F, Na S, Li D, Poh Y-C, Tanaka TS, Wang F, Wang N. Material properties of the cell dictate stress-induced spreading and differentiation in embryonic stem cells. Nat Mater 2010, 9:82-88.

97. Saha S, Ji L, De Pablo JJ, Palecek SP. Inhibition of human embryonic stem cell differentiation by mechanical strain. J Cell Physiol 2006, 206:126-137.

98. Saha S, Ji L, De Pablo JJ, Palecek SP. TGF $\beta$ /activin/ nodal pathway in inhibition of human embryonic stem cell differentiation by mechanical strain. Biophys $J$ 2008, 94:4123-4133.

99. Shimizu N, Yamamoto K, Obi S, Kumagaya S, Masumura T, Shimano Y, Naruse K, Yamashita JK,
Igarashi T, Ando J. Cyclic strain induces mouse embryonic stem cell differentiation into vascular smooth muscle cells by activating PDGF receptor $\beta$. J Appl Physiol 2008, 104:766-772.

100. Heidemann SR, Wirtz D. Towards a regional approach to cell mechanics. Trends Cell Biol 2004, 14:160-166.

101. Gavara N, Roca-Cusachs P, Sunyer R, Farré R, Navajas D. Mapping cell-matrix stresses during stretch reveals inelastic reorganization of the cytoskeleton. Biophys J 2008, 95:464-471.

102. Trepat X, Deng L, An SS, Navajas D, Tschumperlin DJ, Gerthoffer WT, Butler JP, Fredberg JJ. Universal physical responses to stretch in the living cell. Nature 2007, 447:592-595. 